\title{
A Hybrid CFD-DSMC Method of Modeling Continuum-Rarefied Flows
}

\author{
Henry A. Carlson* and Roberto Roveda ${ }^{\dagger}$ \\ Clear Science Corp. \\ Harford, NY 13784-0233 \\ Iain D. Boyd ${ }^{\ddagger}$ \\ Department of Aerospace Engineering \\ University of Michigan, Ann Arbor, MI 48109 \\ Graham V. Candler ${ }^{\S}$ \\ Aerospace Engineering and Mechanics \\ University of Minnesota, Minneapolis, MN 55455
}

\begin{abstract}
A hybrid method of simulating high-speed, non-equilibrium flows solves the NavierStokes equations using a Computational Fluid Dynamics (CFD) model in regions of the flow where properties are continuous and a statistical Direct Simulation Monte Carlo (DSMC) model in regions where properties are non-continuous and thermal nonequilibrium exists. The hybrid method is designed to balance computational efficiency and accuracy by utilizing a relatively inexpensive CFD model whenever appropriate and the DSMC model in critical regions of thermal non-equilibrium. The algorithm uses locally computed values of a breakdown parameter to evaluate the continuum assumption and turns on the DSMC component when the parameter exceeds a threshold value. The DSMC algorithm utilizes the methods of Information Preservation (IP) and ghost cells to accurately and efficiently exchange macroscopic flow properties with the CFD algorithm. Comparisons are made between experimental data for argon flow in a shock tube at Mach numbers 1.55, 5.0, and 10.0 using three computational models-pure CFD, pure DSMC, and hybrid CFD-DSMC. At Mach 1.55, close agreement exists between the experimental data and all of the computational models. At Mach 5 and 10, non-equilibrium becomes significant through the shock layer, and the CFD results diverge from the experimental data. Close agreement exists between the experimental data and both the DSMC and the hybrid simulations at all three Mach numbers, demonstrating that the hybrid method accurately models both equilibrium and non-equilibrium flows. Currently, the hybrid model is more, rather than less, expensive than the pure DSMC model - as a result of limits on the maximum allowable time step size imposed by the IP algorithm. A primary objective of future work is to significantly reduce the computational expense of the hybrid method by incorporating a more robust IP formulation.
\end{abstract}

\section{NOMENCLATURE}

Symbols

Kn Knudsen no.-based breakdown parameter

$M \quad$ Mach number

$N \quad$ Number of simulated molecules

$P \quad$ Breakdown parameter

$Q \quad$ Flow property

$p \quad$ Pressure

$R \quad$ Gas constant

$s \quad$ streamline coordinate

$T \quad$ Temperature

$t \quad$ Time

$t_{p} \quad$ shock thickness

$u_{i} \quad i$-th component of velocity
U Velocity

$x_{i} \quad$ Cartesian coordinate ( $i$-th component)

$\gamma \quad$ Ratio of specific heats

$\lambda \quad$ Mean free path length

$\mu \quad$ Coefficient of viscosity

$\pi \quad$ Circle circumference-to-diameter ratio

$\rho \quad$ Density

$\xi \quad$ Number of molecular degrees of freedom

Subscripts

1 Upstream of shock

2 Downstream of shock

c Macroscopic cell properties

max maximum value

ref Reference value

\footnotetext{
*President, AIAA Associate Fellow

$\dagger$ Research Engineer

‡Professor, AIAA Associate Fellow

$\S$ Professor, AIAA Associate Fellow
} 


\section{INTRODUCTION}

Computation of the aerothermodynamics of hypersonic vehicles along their entire trajectory involves continuum conditions at low altitudes and rarefied conditions at high altitudes. Moreover, continuum and non-continuum conditions often exist simultaneously at a given trajectory point. Examples include shock layers in the forebody region where local gradients are on the order of a mean free path length and afterbody wakes where local Knudsen numbers are an order (or orders) of magnitude higher than the free stream value. Thermal non-equilibrium often exists in these embedded regions, requiring special treatment of the energy balance in the flow, and deviations from equilibrium can affect critical phenomena like aeroheating. ${ }^{1}$

Well-established simulation methods already exist for each of these flow regimes - continuum, equilibrium flow and rarefied, non-equilibrium flow. For example, the continuum Navier-Stokes equations may be solved using algorithms from Computational Fluid Dynamics (CFD) ${ }^{2}$ and the particle-based direct simulation Monte Carlo method (DSMC) ${ }^{3}$ may be used for the rarefied flows. A hybrid method that blends the CFD and DSMC techniques is an attractive approach for flows involving a mixture of continuum and rarefied states.

Target applications include hypersonic flow around launch and reentry vehicles at intermediate altitudes with regions of thermal non-equilibrium embedded in a mostly continuum flow and nozzle-plume problems with continuous flow properties in the nozzle and rarefied conditions in the plume. Chemical vapor deposition, etching, and other material fabrication processes involve flow over a broad range of Knudsen number, and these problems require computational tools like the hybrid CFD-DSMC method. Potential applications extend to the simulation of flow in microelectro-mechanical (MEMS) devices, a rapidly growing business involving hardware that operates over the full range of flow states from continuum to rarefied.

The hybrid CFD-DSMC method has been applied to complex hypersonic flows around cones and cylinderflare configurations ${ }^{4}$ and to unsteady, choked slit flow. ${ }^{6}$ Some of the results have been encouraging, but the complexity of these flows has hampered a clear assessment of the strengths and weaknesses of the method. Here, the algorithm is applied to one-dimensional normal shock waves in argon gas in order to obtain a clearer picture of code performance. The shock tube problem is chosen because 1) it is considerably less expensive computationally than flow problems involving viscous interaction, allowing for high fidelity simulations and 2) comparisons can be made with experimental data of flow property profiles through the shock layer over a range of Mach numbers. ${ }^{11}$ The experimental data were obtained using the electron beam absorption method and have been used to calibrate computational models for more than two decades.

The following two sections contain descriptions of the hybrid algorithm, beginning with the method of identifying continuum breakdown and determining where in the domain the CFD and DSMC models are invoked. This is followed by a description of two methods of filtering the data that is passed between the statistical DSMC model and the CFD model: a physics-based Information Preservation (IP) scheme and ghost cells. In the section following these descriptions of the hybrid method, comparisons are presented between the experimental data and results from CFD, DSMC, and hybrid CFD-DSMC simulations.

\section{DETERMINATION OF CONTINUUM BREAKDOWN}

As flows become rarefied, the velocity distribution deviates from the Maxwellian (equilibrium) distribution, and the Navier-Stokes (NS) equations are no longer valid. A DSMC model is capable of modeling these deviations from equilibrium - but at much greater expense, particularly when the flow is dense. Therefore, the objective in the hybrid method is an algorithm for switching between the CFD and DSMC models that strikes an optimal balance between accuracy and computational efficiency. The algorithm should invoke the relatively expensive DSMC model only in those regions exhibiting non-equilibrium conditions. Bird first proposed a semi-empirical continuum breakdown parameter for steady, expanding flows: ${ }^{12}$

$$
P_{\rho}=\frac{U}{\rho \nu}\left|\frac{d \rho}{d s}\right|=M \sqrt{\frac{\pi \gamma}{8}}\left|\frac{d \rho}{d s}\right|
$$

where $s$ is the distance along a streamline. More recently, a breakdown parameter based on the Chapman-Enskog perturbation expansion of the Boltzmann equation has also been applied to expanding flows. ${ }^{14}$ Bird's parameter may be modified and extended to a more general form by considering multiple flow properties: ${ }^{9,13}$

$$
K n_{Q}=\frac{\lambda}{Q}|\nabla Q|
$$

where $K n$ is the Knudsen number- the ratio of microscopic to macroscopic length scales. From gas kinetics theory, the Knudsen number is a direct measure of the deviation from thermal equilibrium. ${ }^{15}$ The macroscopic length scale is based on a local gradient of flow property $Q$. This length scale in ( 1 ) is based on a projection of the density gradient along a streamline. In ( 2), it is based on the gradient of $Q$ with no projection. When $Q=\rho$, the ratio of the two breakdown parameters defined by ( 1 ) and (2) is

$$
\frac{P_{\rho}}{K n_{\rho}}=M \sqrt{\frac{\pi \gamma}{8}} \cos \theta
$$


where $\theta$ is the angle between the local gradient of $\rho$ and the streamline. Because the local Mach number appears on the right side of $(3), K n_{\rho}$ is orders of magnitude larger than $P_{\rho}$ in subsonic boundary layers and is a more conservative measure of breakdown in those regions.

Both viscous effects and heat transfer contribute to the breakdown of the Navier-Stokes equations in the presence of thermal non-equilibrium. Therefore, the local flow properties that reflect deviation from equilibrium include density, velocity, and temperature. Breakdown parameters that account for the gradients in all three properties are

$$
\begin{aligned}
P_{\max } & =\max \left(P_{\rho}, P_{U}, P_{T}\right) \\
K n_{\max } & =\max \left(K n_{\rho}, K n_{U}, K n_{T}\right)
\end{aligned}
$$

Figure 1 contains results from CFD and DSMC simulations of flow over a sharp cone at Mach 11.3. ${ }^{9}$ Differences between the CFD and DSMC solutions reflect deviations from continuum, and these differences are quantified as

$$
\epsilon_{Q}=\frac{Q_{C F D}}{Q_{D S M C}}-1
$$

Failure of the continuum assumption is defined as occurring when $\left|\epsilon_{Q}\right|>0.05$. The horizontal axes in Figure 1 are the distance from the cone surface along a line normal to the axis of symmetry, located aft of the tip. From the top panel, the variation in Mach number indicates that CFD predicts a much thinner shock layer than DSMC, reflecting non-equilibrium conditions in the shock layer, undetected by CFD. $\epsilon_{\rho, T}$ are greater than the threshold values of \pm 0.05 (demarked by the dotted horizontal lines) through the shock layer; $\epsilon_{U}$ is not. In the boundary layer, $\epsilon_{\rho, U, T}$ are all above the threshold value. Using a threshold value of 0.05 for the breakdown parameter as well, a comparison of the top and bottom panels of Figure 1 indicates that $K n_{\text {max }}$ (DSMC) is a good measure of non-equilibrium, defined as a breakdown in the CFD model.

However, the purpose of the hybrid method is to provide accurate predictions at a computational cost that is lower than the cost of DSMC. Therefore, a CFD (not a DSMC) solution will provide the initial conditions for hybrid simulations. The compromise between accuracy and expense is to use a conservative (i.e. small) cut-off value of the breakdown parameter and the CFD solution in determining where in the domain to turn on the DSMC component of the hybrid code. This approach will be taken in the shock tube tests.

\section{CFD-DSMC MODEL INTERFACES}

The second objective in the hybrid CFD-DSMC method is an accurate and consistent exchange of flow property values at the interfaces between regions in
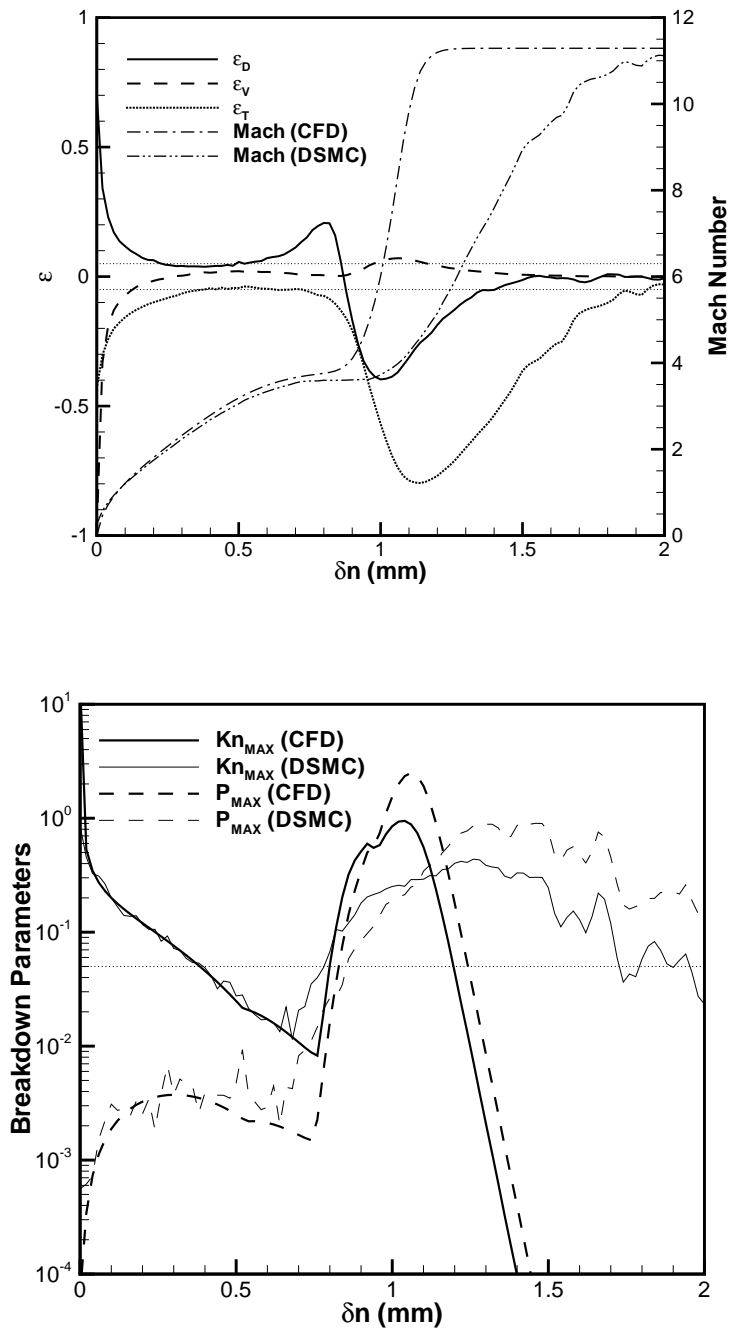

Fig. 1 From Wang and Boyd. ${ }^{9}$ Comparison of CFD and DSMC simulations of flow around a sharp cone at Mach 11.3: density, velocity, temperature, and Mach number (top) and breakdown parameters (bottom).

the flow where the CFD model is applied and regions where the DSMC model is applied. The continuum and kinetic algorithms exchange information based on the evaluation (and extrapolation) of either macroscopic properties or fluxes near the interface. An important issue in a coupled hybrid scheme is control of the statistical scatter in the DSMC signal to provide smooth boundary conditions for the continuum solver. In a weakly coupled scheme for steady flows, the scatter level is controllable. It is possible to cycle the CFD and DSMC solutions separately in order to obtain an adequate number of samples for the averaging of macroscopic properties. In a strongly coupled problem involving the exchange of information at every time step, extensive averaging and smoothing procedures are not possible. For these types of prob- 
lems, Roveda et al developed the method of "ghost levels". 5,6

To reduce the statistical scatter in the flow properties properties passed from the DSMC model even more, the hybrid CFD-DSMC algorithm employs the Information Preservation (IP) method, which preserves macroscopic information throughout the DSMC portion of the domain. ${ }^{7,8}$ The preserved information is updated at each time step with both collisional and pressure-based data and is available to the CFD model at all locations in the domain. Macroscopic values of velocity and temperature are functions of local pressures in addition to momentum and energy exchange. Therefore, the process requires two operations: 1) collision-move algorithms are applied to the simulated molecules and are used to update local, macroscopic properties and 2) the macroscopic properties are then modified to include pressure effects. An additional energy transfer term is required to reconcile a difference between the IP representation of energy flux across a cell interface and the actual energy transfer. The average translational energy of a molecule at equilibrium temperature $T$ is $3 k T / 2$ where $k$ is the Boltzmann constant. The average translational energy carried by a molecule across a cell interface is $2 k T$. The difference in these values is accounted for by assigning an additional term $\left(T_{a}\right)$ to the molecular temperatures that determine macroscopic properties.

The preserved velocity and temperature are updated by solving

$$
\frac{\partial u_{i}}{\partial t}=-\frac{1}{\rho_{c}} \frac{\partial p_{c}}{\partial x_{i}}
$$

and

$$
\frac{\partial}{\partial t}\left(\frac{u_{i} u_{i}}{2}+\frac{\xi R T}{2}\right)=-\frac{1}{\rho_{c}} \frac{\partial}{\partial x_{i}}\left(u_{i, c} p_{c}\right)
$$

where $p, \rho, T, u_{i}, R$, and $\xi$ are pressure, density, temperature, molecular velocity, specific gas constant, and number of molecular degrees of freedom, respectively. Terms with the subscript $c$ are macroscopic properties assigned to a computational cell. Values of $T_{c}$ and $u_{i, c}$ are updated by sampling molecular properties within a computational cell:

$$
u_{i, c}=\frac{1}{N} \sum_{n=1}^{N} u_{i, n} \quad ; \quad T_{c}=\frac{1}{N} \sum_{n=1}^{N}\left(T_{n}+T_{a, j}\right) .
$$

Density and pressure are updated using the relation for conservation of mass and the ideal gas law:

$$
\frac{\partial \rho_{c}}{\partial t}=-\frac{\partial}{\partial x_{i}}\left(\rho_{c} u_{i, c}\right) \quad ; \quad p_{c}=\rho_{c} R T_{c} .
$$

Figure 2 is a schematic of the CFD/DSMC-IP interface. There are two levels of ghost cells: buffer and reservoir. At each time step, molecules from the DSMC domain that end their movement within the
DSMC domain (the buffer) are retained. Molecules that reside in the reservoir at the end of each time step are first deleted and then re-generated with properties computed from the Chapman-Enskog distribution based on cell-centered values. The re-generated molecules are randomly distributed within the reservoir cells. Because macroscopic DSMC-IP properties are updated at each time step, the DSMC-IP cells adjacent to the interface are treated as ghost cells and provide the boundary conditions for flux computations. Therefore, in the CFD domain, the CFD model computes continuum fluxes from the CFD-model variables and the macroscopic properties from adjacent DSMC-IP cells.
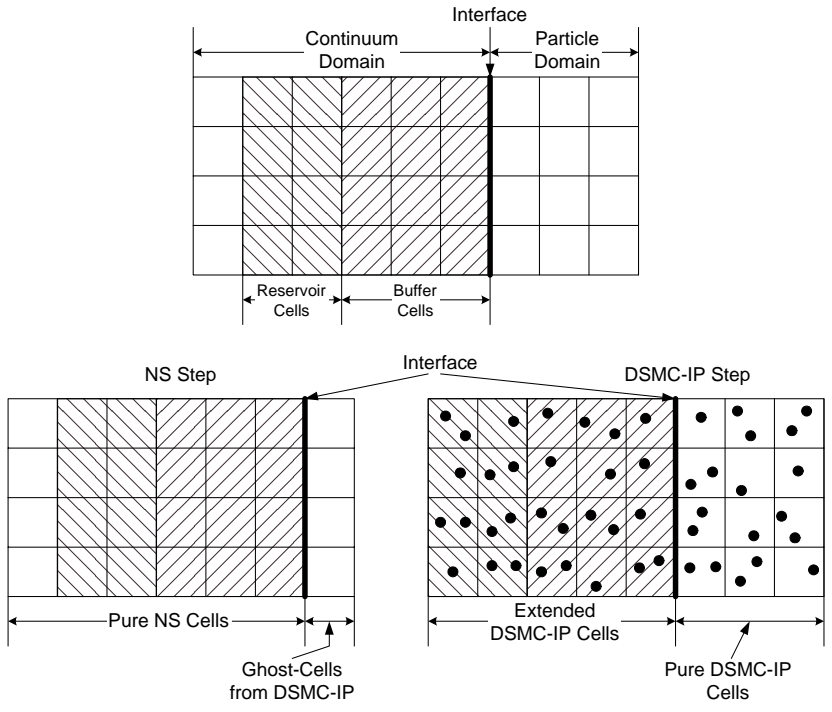

Fig. 2 From Wang et al. ${ }^{10}$ Schematic of the CFDDSMC interface, which includes reservoir (ghost) cells and DSMC-IP cells to reduce the effects of statistical noise.

\section{RESULTS}

The test consists of Argon flow in a shock tube at Mach 1.55, 5, and 10 with upstream temperature and pressure of $300 \mathrm{~K}$ and $50 \mathrm{mTorr}$, respectively. Comparisons are made between the experimental data of Alsmeyer ${ }^{11}$ and results from three computational models: CFD, DSMC, and hybrid CFD-DSMC. The pure CFD model and the CFD component of the hybrid model consist of explicit algorithms that solve the full Navier-Stokes equations. In all of the simulations, the mesh consists of 400 by 5 grid points. The pure DSMC model employs approximately 200,000 simulated molecules with 50,000 iterations to steady state, ensuring more than adequate spatial resolution and statistical fidelity. The CFD viscosity model is

$$
\mu=\mu_{\text {ref }}\left(\frac{T}{T_{\text {ref }}}\right)^{0.75}
$$


where $\mu_{\text {ref }}=2.1 \times 10^{-5} \mathrm{~N}-\mathrm{s} / \mathrm{m}^{2}$ is the reference viscosity of argon at $T_{\text {ref }}=288 \mathrm{~K}$. The DSMC collision model parameters are consistent with the CFD viscosity model.

The hybrid code uses the pure CFD solution to compute the breakdown parameters and determine CFDDSMC interfaces. In these tests, the breakdown cut-off value is set (conservatively) to 0.005 . The CFD solution is also used to initialize the hybrid solution. At all three Mach numbers, the shock wave moves slightly within the computational domains of all three models. To facilitate comparison of the model results, the shock positions are translated such that the normalized density value of 0.5 is located at $x_{1}=0$. In all of the test results, density and temperature are normalized as

$$
\rho_{n}=\frac{\rho-\rho_{1}}{\rho_{2}-\rho_{1}} \quad ; \quad T_{n}=\frac{T-T_{1}}{T_{2}-T_{1}}
$$

where $\rho_{1}, T_{1}$ and $\rho_{2}, T_{2}$ correspond to conditions upand downstream of the shock, respectively. Therefore, normalized values are zero upstream of the shock and unity downstream.

In the original experiments, Alsmeyer compared DSMC results from Bird and reported excellent agreement with the experimental data at all Mach numbers. ${ }^{11}$ Because the work was performed more than 25 years ago, comprehensive, direct graphical comparison of current simulation results and the experimental data is not practicable. In the following three figures, comparisons are made between the current CFD, DSMC, and hybrid model results with the assumption that the current DSMC results are highly accurate. Visual inspection of the experimental and DSMC data reported by Alsmeyer and the current DSMC data support this assumption. The final figure is a direct comparison of the original experimental data and all of the current simulation results, providing additional confirmation of the assumption.

Figure 3 contains normalized profiles of density and temperature through the Mach 1.55 shock wave. At this relatively low Mach number, the degree of nonequilibrium in the shock is slight, and the NavierStokes equations provide a reasonably accurate solution of the flow. Therefore, the CFD and DSMC results are in good agreement. The "Hybrid" symbols in Figure 3 correspond to data from the DSMC component of the hybrid code through the shock layer. This corresponds to the region labeled "particle" $(-10 \geq x / \lambda \leq+8.5) . x / \lambda=-10$ and $x / \lambda=+8.5$ are interfaces between the CFD and DSMC components of the hybrid code. Flow properties upstream of the shock at $x / \lambda=-10$ are passed from CFD to DSMC, and properties downstream of the shock at $x / \lambda=+8.5$ are passed from DSMC to CFD. The flow properties computed by the CFD and DSMC components of the hybrid code are in close agreement at the two interfaces, demonstrating that the hybrid method accurately communicates information across the continuum-particle boundaries.
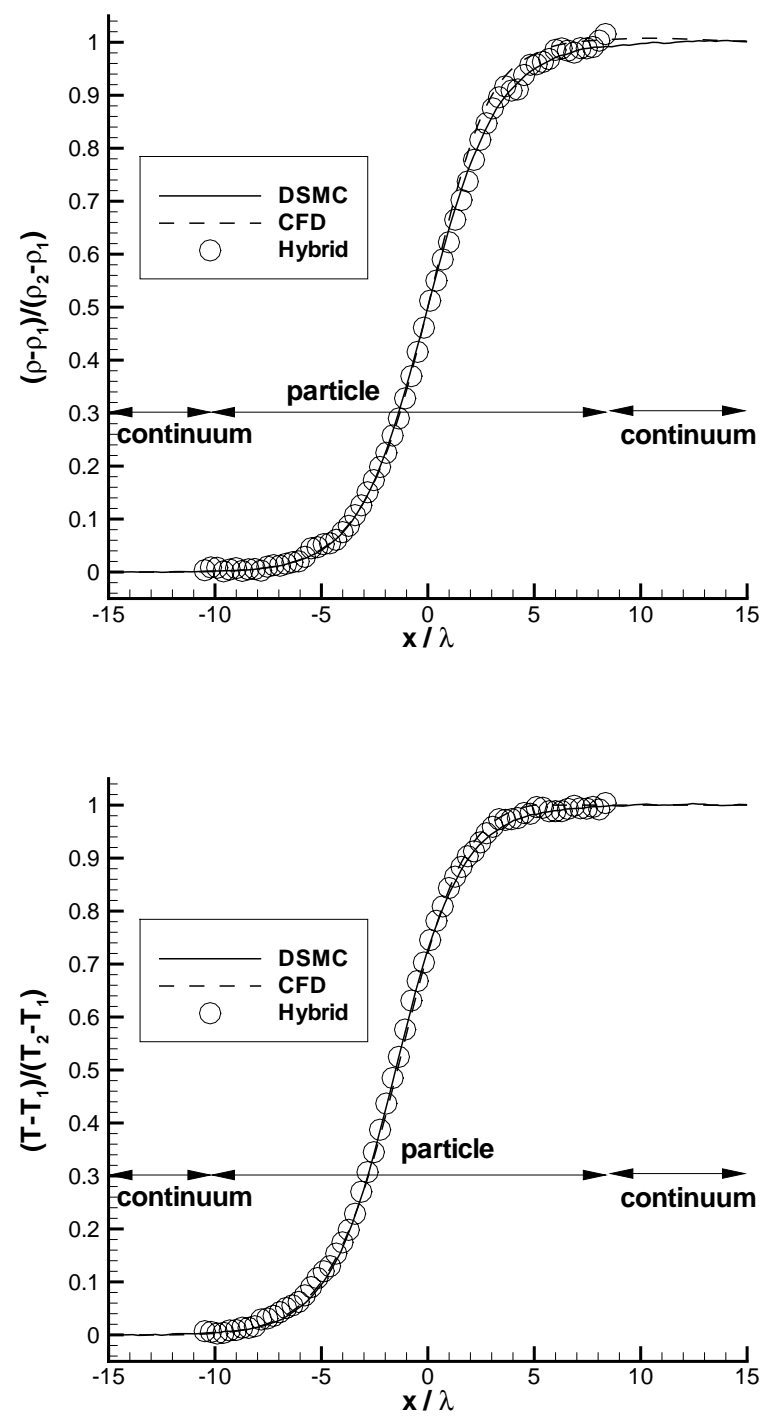

Fig. 3 Profiles of normalized density (top) and temperature (bottom) through a $M=1.55$ normal shock wave of argon.

Figure 4 contains normalized profiles of density and temperature through the Mach 5 shock wave. This case represents a much more difficult condition for the CFD method, because a significant deviation from thermal equilibrium occurs in the shock. Comparison of the CFD and DSMC results indicates that the CFD model predicts a significantly thinner shock layer than the DSMC model. The hybrid results demonstrate that the method is successful in moving the solution from the initial, incorrect (CFD) solution inside the shock to the correct (DSMC) solution.

Figure 5 contains normalized profiles of density and temperature through the Mach 10 shock wave. Again, 

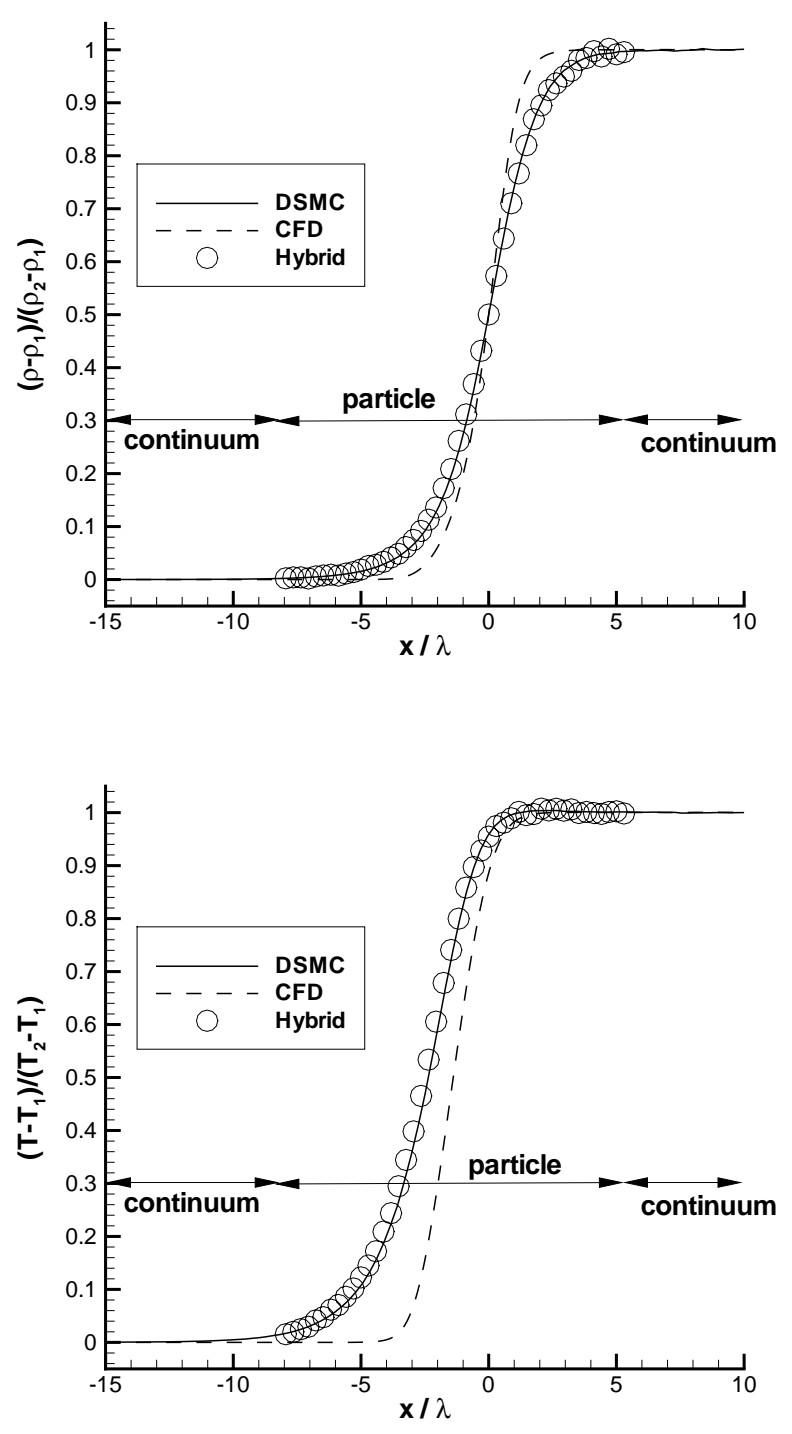

Fig. 4 Profiles of normalized density (top) and temperature (bottom) through a $M=5$ normal shock wave of argon.

the CFD under-predicts the shock thickness by a significant amount, and, again, the hybrid model begins with this CFD solution and moves it to the much thicker DSMC profile.

To test the assumed accuracy of the DSMC model (and the relative accuracies of the CFD and hybrid models), reciprocal shock thicknesses are computedat a number of additional Mach numbers using the CFD and DSMC models and at the original three Mach numbers using the hybrid model - and direct comparisons are made with the experimental data of Alsmeyer. The reciprocal shock thickness is based on the normalized density gradient at the center of the
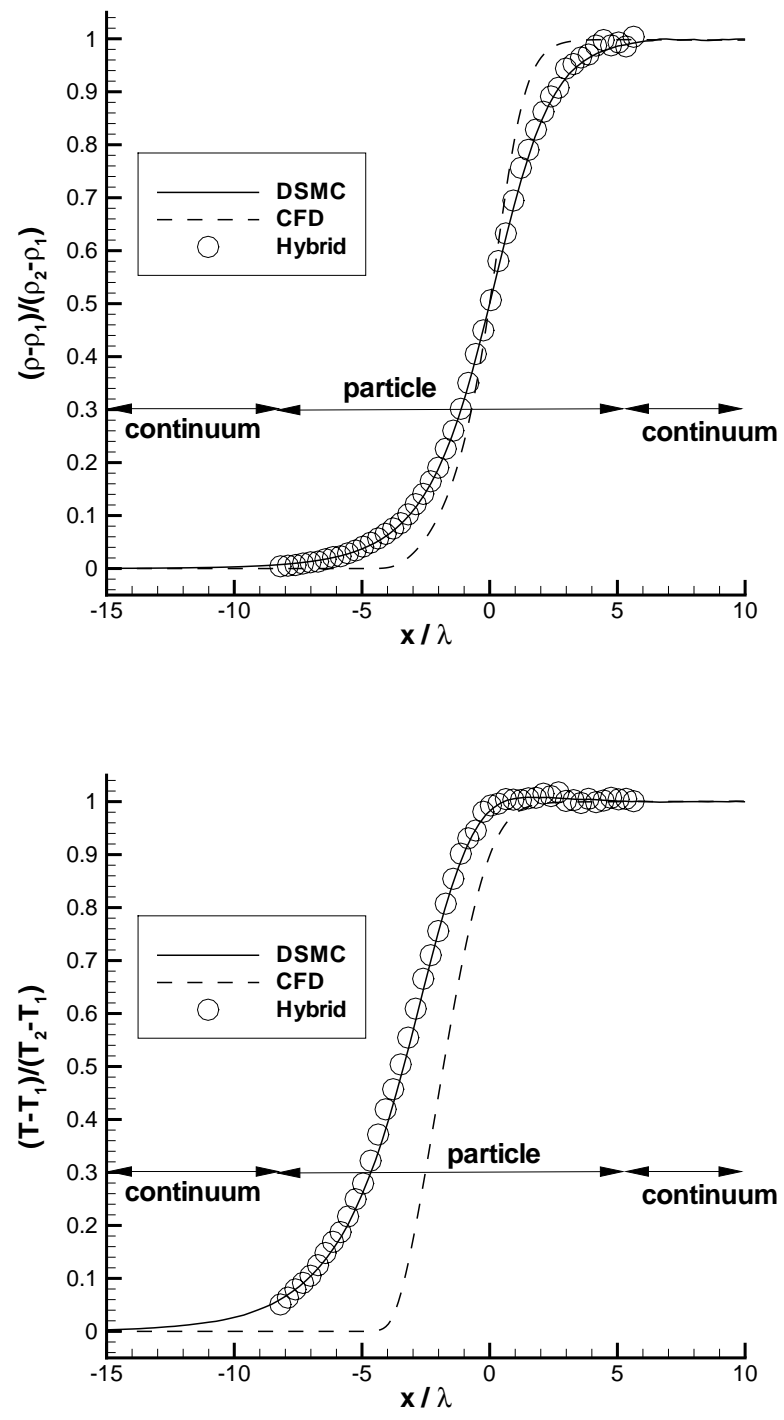

Fig. 5 Profiles of normalized density (top) and temperature (bottom) through a $M=10$ normal shock wave of argon.

$\operatorname{shock}\left(x_{1} / \lambda_{1}=0\right)$ :

$$
t_{p}^{-1}=\left.\frac{1}{\rho} \frac{\partial \rho}{\partial x_{1}}\right|_{x_{1} / \lambda_{1}=0}
$$

From Figure 6, the DSMC model is in excellent agreement with the experimental data across the entire range of Mach number. The CFD model is in agreement to approximately Mach 1.5 where it begins over-predicting the reciprocal thickness by a significant amount. Most importantly, the hybrid model is in close agreement with the experimental data and DSMC model at the three Mach numbers tested, clearly providing significantly more accuracy than the CFD solutions that were used to initialize the runs.

Finally, the computational cost of the hybrid model 


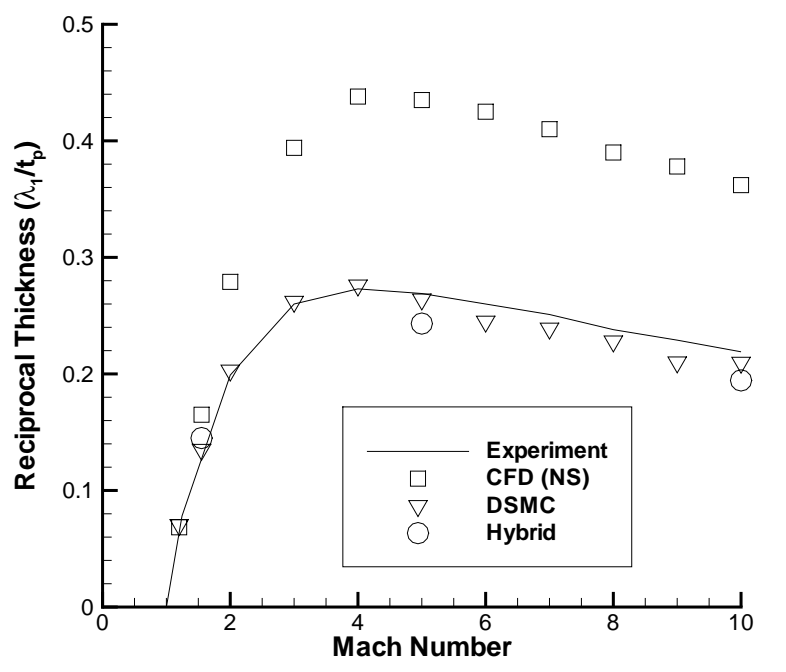

Fig. 6 Reciprocal shock thicknesses for normal shock waves of argon. Experimental data from Alsmeyer. ${ }^{11}$

\begin{tabular}{lcc}
\hline \hline Method & Total CPU time & CPU time per step \\
\hline CFD & 1.00 & 1.00 \\
DSMC & 10.7 & 15.0 \\
Hybrid & 16.7 & 9.06 \\
\hline \hline
\end{tabular}

Table 1 Computational costs: CPU times for the Mach 5 runs, normalized by the CFD CPU time.

is measured relative to the CFD and DSMC models. In Table 1, total CPU times for the Mach 5 runs are normalized by the CPU time of the CFD model. The DSMC model total time is an order of magnitude higher than the CFD time, reflecting the additional overhead attached to accurately modeling non-equilibrium flows. The CFD formulation is explicit. With an implicit formulation, the ratio of total CFD time to DSMC time would be smaller, particularly for problems involving viscous interaction. The hybrid model total time is more than $50 \%$ higher than the DSMC time, which clearly does not meet one of the stated objectives: the accuracy of the hybrid model is vastly superior to the CFD model, but the cost is not less than DSMC.

The computational overhead of the IP component of the hybrid model is approximately $30 \%$. That is, the DSMC-IP algorithm consumes 1.3 times more CPU time than the DSMC algorithm. However, the most significant increase in overhead occurs because the IP algorithm reduces the maximum time step size in the hybrid simulation to $1 / 20$ th of the maximum allowable DSMC time step size. Therefore, the number of time steps required with the hybrid model is 20 times greater than the number required with DSMC. This more than offsets the saving gained by computing with CFD in a portion of the domain. Increasing the maximum time step size in the hybrid model to sizes currently achievable with DSMC can make the hybrid formulation significantly less expensive than DSMCnotwithstanding the $30 \%$ overhead of IP - and this is a primary objective of future work. The second column of Table 1 contains normalized CPU times per time step and reflects a 50\% speed-up in the hybrid method over DSMC when the time step sizes are identical.

\section{CONCLUSIONS}

A hybrid CFD-DSMC method has been developed to simulate hypersonic, non-equilibrium flows. The method may be applied to flow states ranging from continuum to rarefied. The hybrid model identifies regions of non-equilibrium by computing a continuum breakdown parameter based on local mean free path length and local gradients of density, temperature, and velocity. The CFD component of the model is applied in regions where the parameter is below a threshold value, and the DSMC component is applied in all other regions. The methods of ghost levels and Information Preservation (IP) are applied at interface boundaries, ensuring that flow properties are accurately passed between the CFD and DSMC components in a consistent manner. Comparisons with experimental data of shock tube flow demonstrate that the hybrid model accurately predicts shock thicknesses over a broad range of Mach number in both equilibrium and non-equilibrium shock layers. Currently, the hybrid model is more, rather than less, expensive than the pure DSMC model - as a result of limits on the maximum allowable time step size imposed by the IP algorithm. A primary objective of future work is to significantly reduce the computational expense of the hybrid method by incorporating a more robust IP formulation.

\section{ACKNOWLEDGMENTS}

This material is based upon work supported by the Air Force Research Laboratory (Computational Sciences Branch of the Aeronautical Sciences DivisionAFRL/VAAC), located at Wright-Patterson Air Force Base, under Contract F33615-03-M-3330. Any opinions, findings, conclusions, or recommendations expressed in this material are those of the authors and do not necessarily reflect the views of the Air Force Research Laboratory. Technical contributions to this study made by Wen-Lan Wang are gratefully acknowledged.

\section{References}

${ }^{1}$ Carlson, H. A. , "Aerothermodynamic Analyses of Hypersonic, Blunt-Body Flows," J. Spacecr. Rockets, 36, No. 6, 1999, pp. 912-915.

${ }^{2}$ MacCormack, R. W., and Candler, G. V., "The Solution of the Navier-Stokes Equations Using Gauss-Seidel Relaxation," Computers and Fluids, Vol. 17, 1989, pp. 135-150. 
${ }^{3}$ Bird, G. A., Molecular Gas Dynamics and the Direct Simulation of Gas Flows, Oxford University Press, 1994.

${ }^{4}$ Wang, W.-L. and Boyd, I. D., "Hybrid DSMC-CFD Simulations of Hypersonic Flow Over Sharp and Blunted Bodies," AIAA Paper 2003-3644, June 2003.

${ }^{5}$ Roveda, R. , Goldstein, D. B. , and Varghese, P. L. , "A Combined Discrete Velocity/Particle Based Numerical Approach for Continuum/Rarefied Flows," AIAA paper 97-1006, Jan. 1997.

${ }^{6}$ Roveda, R. , Goldstein, D. B. , and Varghese, P. L , "Hybrid Euler/Particle Approach for Continuum/Rarefied Flows," J. Spacecr. Rockets, 35, No. 3, 258 (1998).

${ }^{7}$ Fan, J. and Shen, C. , "Statistical Simulation of LowSpeed Unidirectional Flows in Transition Regime," in Rarefied Gas Dynamics, Vol. 2, ed. R. Brun et al.,(Cepadus-Editions, Toulouse), 1999, p. 245.

${ }^{8}$ Sun, Q. and Boyd, I. D., "A Direct Simulation Method for Subsonic, Microscale Gas Flows," Journal of Computational Physics, Vol. 179, 2002, pp. 400-425.

${ }^{9}$ Wang, W.-L. and Boyd, I. D., "Predicting Continuum Breakdown in Hypersonic Viscous Flows," Physics of Fluids, Vol. 15, 2003, pp. 91-100.

${ }^{10}$ Wang, W. L. , Sun, Q. , and Boyd, I. D. , "Towards Development of a Hybrid DSMC-CFD Method for Simulating Hypersonic Interacting Flows," AIAA Paper 2002-3099, 2002.

${ }^{11}$ Alsmeyer, H., "Density Profiles in Argon and Nitrogen Shock Waves Measured by the Absorption of an Electron Beam," Journal of Fluid Mechanics, Vol. 74, 1976, pp. 497-523.

${ }^{12}$ Bird, G. A. , "Breakdown of Translational and Rotational Equilibrium in Gaseous Expansions," AIAA J., 8, 1970, pp. 1998-2003.

${ }^{13}$ Boyd, I. D. , Chen, G. , and Candler, G. V. , "Predicting Failure of the Continuum Fluid Equations in Transitional Hypersonic Flows," Phy. Fluids, 7, No. 1, 1995, pp. 210-219.

${ }^{14}$ George, J. D. and Boyd I. D. , "Simulation of Nozzle Plume Flows Using a Combined CFD-DSMC Approach," AIAA Paper 1999-3454, 1999.

${ }^{15}$ Gombosi, T. I. , Gaskinetic Theory, Cambridge University Press, 1994 